\title{
Shear-Regulated Extracellular Microenvironments and Endothelial Cell Surface Integrin Receptors Intertwine in Atherosclerosis
}

\author{
Fan- $E M o^{1,2 *}$ \\ ${ }^{1}$ Department of Cell Biology and Anatomy, College of Medicine, National Cheng Kung University, Tainan, Taiwan, ${ }^{2}$ Institute of \\ Basic Medical Sciences, College of Medicine, National Cheng Kung University, Tainan, Taiwan
}

Mechanical forces imposed by blood flow shear stress directly modulate endothelial gene expression and functional phenotype. The production of extracellular matrix proteins and corresponding cell-surface integrin receptors in arterial endothelial cells is intricately regulated by blood flow patterns. Laminar blood flow promotes mature and atheroresistant endothelial phenotype, while disturbed flow induces dysfunctional and atheroprone endothelial responses. Here, we discuss how hemodynamic changes orchestrate the remodeling of extracellular microenvironments and the expression profile of the integrin

OPEN ACCESS

Edited by:

Jing Zhou,

Peking University, China

Reviewed by:

Orest William Blaschuk,

McGill University, Canada

Myeongwoo Lee,

Baylor University, United States

*Correspondence:

Fan-EMo

femo@mail.ncku.edu.tw;

fanemo@gmail.com

Specialty section:

This article was submitted to Cell Adhesion and Migration,

a section of the journal

Frontiers in Cell and Developmental

Biology

Received: 12 December 2020 Accepted: 18 March 2021

Published: 06 April 2021

Citation:

Mo F-E (2021) Shear-Regulated Extracellular Microenvironments and

Endothelial Cell Surface Integrin

Receptors Intertwine in

Atherosclerosis.

Front. Cell Dev. Biol. 9:640781.

doi: 10.3389/fcell.2021.640781 receptors in endothelial cells leading to oxidative stress and inflammation. Targeting the interaction between matrix proteins and their corresponding integrins is a potential therapeutic approach for atherosclerosis.

Keywords: disturbed flow atherosclerosis, extracellular matrix, integrin, matricellular proteins, endothelial dysfunction, atheroprotection

\section{INTRODUCTION}

Though risk factors, such as hyperlipidemia, hypertension, and hyperglycemia, pose a threat to the entire arterial system, atherosclerosis preferentially occurs at arterial branches or curvatures, where the local blood flow is disturbed (Chiu and Chien, 2011). Blood flow imposes fictional drag, called sheer stress, directly to the inner lining endothelial cells (ECs). Laminar blood flow found at the straight part of artery generates unidirectional shear stress and promotes functional endothelial phenotype (atheroprotective). By contrast, disturbed flow generates low and oscillatory sheer stress, and induces EC activation and maladaptive alterations in endothelial functional phenotype (atheroprone; Hsu et al., 2019). Low endothelial shear stress correlates with increased plaque burden and risks of rupture in patients with coronary artery disease (Chatzizisis et al., 2008; Stone et al., 2012). Endothelial dysfunction by disturbed flow is manifested by the lack of nitric oxide production, and chronic inflammatory response mediated through the pleiotropic transcription factor nuclear factor- $\mathrm{kB}$ (NF- $\mathrm{kB}$; Gimbrone and Garcia-Cardena, 2016). These atheroprone genes include cell-surface adhesion molecules (such as vascular cell adhesion molecule-1; Korenaga et al., 1997), secreted cytokines (such as interleukin-1 and monocyte chemoattractant protein 1; Hsu et al., 2019), and prothrombotic mediators (such as von Willebrand factor; Zhu et al., 2020). Recent studies using systems biology approaches have demonstrated that low endothelial shear stress causes aberrant reactivation of vascular developmental signaling pathways, such as BMP-TGF $\beta$, WNT, Notch, HIF1 $\alpha$, TWIST1, and HOX family genes, leading to increased inflammation and vascular permeability (Souilhol et al., 2020). 
Consequently, the infiltration of monocytes/macrophages rises, the uptake of lipid elevates, local inflammation and oxidative stress intensifies, and the number of vascular smooth muscle cells increases in the intima, leading to the formation of atherosclerotic plaques. Emerging evidence suggests that local microenvironment also plays a major role in regulating endothelial cell function and regional susceptibility to atherosclerosis (Yurdagul et al., 2016). Hemodynamics may affect endothelial remodeling and change subendothelial matrix composition. Concurrently, the expression profile of cell-surface integrin receptors responsible for interacting with the extracellular matrix (ECM) is adjusted in the ECs to accommodate the changing microenvironment. The signaling induced by the engagement of ECM proteins and corresponding integrin receptors regulates endothelial cell functional phenotype. The critical role in atherogenesis and the accessibility of matrix/ integrin engagement makes it an attractive therapeutic target for atherosclerosis. This minireview summarizes recent findings on the shear-induced ECM remodeling and integrin expression, and their roles in atherosclerosis (Figure 1).

\section{BASEMENT MEMBRANE}

The basement membrane is the subendothelial ECM largely secreted by and closely interacting with ECs. The major components of the basement membrane in normal vessels include laminin and type IV collagen (Yurchenco and O'Rear, 1994). Both laminin and collagen IV promote homeostasis of ECs, and prevent disturbed flow-induced NF- $\mathrm{kB}$ activation and inflammation (Orr et al., 2005). The composition of basement membrane is regulated by hemodynamic patterns. Disturbed flow induces subendothelial matrix remodeling to a "provisional" matrix rich in fibronectin at atheroprone sites prior to other signs of atherosclerosis (Orr et al., 2005). The provisional ECM is initially named with regard to the transient ECM (enriched with fibronectin, vitronectin, and fibrinogen) that is deposited and subsequently cleared during wound healing (Stupack and Cheresh, 2002). A fibronectin-rich ECM primes ECs to activation by oxidized low-density lipoprotein (Yurdagul et al., 2014) and hyperglycemia (Green et al., 2014). Onset of atheroprotective laminar shear stress induces a transient fibronectin upregulation compared to static control in vitro, whereas atheroprone oscillatory shear stress initiates a steady increase in fibronectin expression through the activation of NF- $\mathrm{\kappa B}$. Furthermore, because fibronectin promotes NF- $\kappa B$ activation, disturbed flow creates positive feedback to sustain endothelial inflammation (Feaver et al., 2010). Whereas disturbed shear activates p21-activated kinase in ECs on fibronectin leading to enhanced $\mathrm{NF}-\mathrm{\kappa B}$ and inflammation, ECs on native basement proteins inhibits shear-induced p21-activated kinase activation through a protein kinase A-dependent pathway.

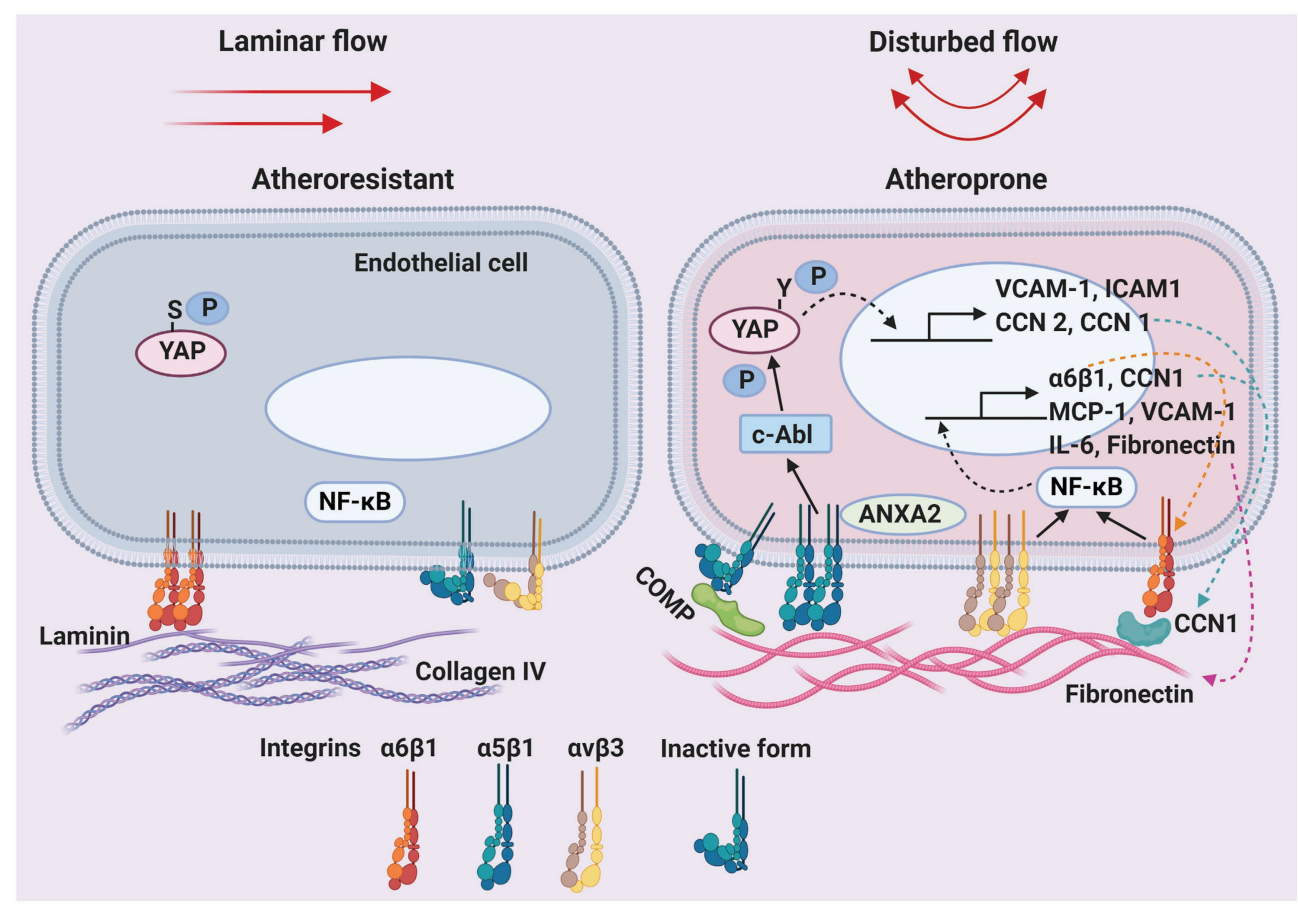

FIGURE 1 | Shear-regulated extracellular microenvironments and endothelial integrin receptors in atherosclerosis. Schematic of the dynamic changes in the composition of the endothelial basement membrane and cell-surface integrin receptors by unidirectional laminar blood flow or oscillatory disturbed flow. Solid arrows: activation of downstream effectors. Dashed arrows: translocation of proteins. ANXA2, annexin A2; COMP, cartilage oligomeric matrix protein; ICAM1, intercellular adhesion molecule 1; IL-6, interleukin-6; MCP-1, monocyte chemoattractant protein 1; NF-кB, nuclear factor-кB; P, phosphorylation; S, serine; VCAM-1, vascular cell adhesion molecule 1; Y, tyrosine; YAP, yes-associated protein. 


\section{INTEGRINS}

Integrins are transmembrane heterodimeric proteins consisting of $\alpha$ and $\beta$ subunits, responsible for cell adhesion to ECM and transducing environmental cues and internal cell signals in both directions. There are at least $18 \alpha$ subunits and eight $\beta$ subunits through different combinations to form 24 distinct integrins (Hynes, 2002). ECs express a repertoire of integrins to mediate dynamic interactions between cells and ECM. Endothelium on a native ECM expresses the collagen-binding ( $\alpha 1 \beta 1$ and $\alpha 2 \beta 1)$ and laminin-binding ( $\alpha 3 \beta 1, \alpha 6 \beta 1$, and $\alpha 6 \beta 4)$ integrins for mediating EC anchorage within quiescent blood vessels. ECs on a provisional matrix express RGD-binding integrins (including $\alpha 5 \beta 1$ and $\alpha v \beta 3$ ), resulting in EC activation for angiogenesis or in pathologic conditions (Stupack and Cheresh, 2002). $\alpha v \beta 3$ critically mediates EC interactions with provisional matrix proteins and disturbed flow-induced pro-inflammatory signaling (NF- $\mathrm{kB}$ and p21-activated kinase activation) in early atherogenic stages. However, inhibiting $\alpha v \beta 3$ does not affect all the shear-induced signaling, as Akt, endothelial nitric oxide synthase, and extracellular regulated kinase remain responsive (Chen et al., 2015). Endothelial $\alpha 5 \beta 1$ binding with fibronectin under low oscillatory shear stress phosphorylates (activates) the cytosolic nonreceptor protein kinase c-Abl, which then induces tyrosine phosphorylation (at $\mathrm{Y}^{357}$ ) and nuclear translocation of yes-associated protein (YAP), leading to pro-atherogenic gene expression and EC activation. The phosphorylation of $\mathrm{c}-\mathrm{Abl}$ and $\mathrm{YAP}^{\mathrm{Y} 357}$ is significantly higher in atherosclerotic plaques of $A p o e^{-/-}$mice and in patients (Li et al., 2019). Of note, YAP can also be serine phosphorylated at S127 (for 14-3-3 binding and cytoplasmic retention) and at S381 (for ubiquitination and degradation), thus inactivated by the Hippo pathway (Yu et al., 2015). As such, unidirectional shear flow sustains the cytoplasmic levels of YAP Y127,\$381 $^{\$}$ phosphorylation and suppression in ECs for atheroprotection through RhoA inhibition (Wang et al., 2016b). Endothelial $\alpha 5 \beta 1$ upon binding with fibronectin also recruits phosphodiesterase 4D5 at adhesion sites, which subsequently connects with phosphatase PP2A and its regulatory subunit B55 $\alpha$. Fibronectininduced PP2A holoenzyme assembly triggers $\mathrm{YAP}^{\mathrm{S} 127}$ dephosphorylation and facilitates YAP activation (Yun et al., 2019). Although no direct evidence, increased levels of YAP by disturbed flow in ECs is consistent with the possibility of YAP ${ }^{5381}$-dephosphorylation (lower ubiquitination and degradation; Wang et al., 2016a). Furthermore, to effectively transduce outside-in signaling, inactive integrins require inside-out signaling to make a conformational change and promote their binding with permissive ligands. Disturbed flow activates endothelial $\alpha 5 \beta 1$ by increasing calcium influx through the cation channel Piezo1, which activates PTP1B-dependent dephosphorylation of annexin A2. Annexin A2 then binds to integrin $\alpha 5$ and facilitates translocation to lipid rafts to induce integrin activation and ligation (Zhang et al., 2020). Integrin $\alpha 5 \beta 1$ activation is essential for fibronectin deposition and proinflammatory responses by atheroprone shear or oxidized low-density-lipoproteins (Yurdagul et al., 2014; Al-Yafeai et al., 2018). Interestingly, laminin-binding integrin $\alpha 6 \beta 1$ is also induced by disturbed flow (Hsu et al., 2019). $\alpha 6 \beta 1$ binds to other ECM molecules in addition to laminin, which will be discussed below.

\section{MATRICELLULAR PROTEINS}

Matricellular proteins are a group of extracellular proteins that do not directly serve structural roles, but rather function contextually as regulators of cell-matrix interactions and cell function (Bornstein and Sage, 2002). A number of matricellular proteins have been implicated in atherosclerosis. Among them, CCN1/CYR61 is the most well-characterized example of how matricellular proteins may regulate the formation of atherosclerosis. CCN1, a member of the CCN matricellular protein family, binds to at least seven integrin receptors, including $\alpha 6 \beta 1$ and $\alpha v \beta 3$ on ECs (Kireeva et al., 1998; Leu et al., 2003; Chen and Lau, 2009), thus regulating diverse cellular activities including cell adhesion, migration, differentiation, proliferation, and survival/apoptosis/senescence (Lau, 2016). CCN1 is expressed in atherosclerotic lesions in patients (Hilfiker et al., 2002) and in the Apoe ${ }^{-1-}$ mouse models (Hsu et al., 2019). CCN1 expression is upregulated by oscillatory shear stress, and downregulated by laminar shear stress in ECs (Wang et al., 2016a,b; Hsu et al., 2019). Shear-induced CCN1 binds to $\alpha 6 \beta 1$ and causes atheroprone phenotypic changes in EC via NF- $\kappa \mathrm{B}$ activation, which further increases the expression of CCN1, $\alpha 6$, and $\beta 1$ in a vicious circle. A peptide antagonist selectively targeting CCN1$\alpha 6 \beta 1$ engagement has been tested and effectively inhibits disturbed flow-induced atherogenesis in vitro and in vivo (Hsu et al., 2019). $\alpha 6 \beta 1$ is expressed at basal levels in quiescent ECs and binds to basement membrane protein laminin at focal adhesion sites (Seano et al., 2014). A fibronectin-rich provisional matrix under disturbed flow releases $\alpha 6 \beta 1$ from focal adhesions (Chen et al., 2015). The combination of $\alpha 6 \beta 1$ upregulation and disassembly by disturbed flow results in more accessible receptor integrins for $\mathrm{CCN} 1$ action.

CCN2/connective tissue growth factor and CCN1 share similar integrin receptors and are co-expressed in advanced atherosclerotic lesions in mice (Schober et al., 2002). Both CCN1 and CCN2 are directly induced by YAP/TAZ in ECs under oscillatory shear stress (Wang et al., 2016a,b). CCN2 has been demonstrated to increase neointimal thickening in a rat carotid artery angioplasty model (Kundi et al., 2009). However, the role of CCN2 in atherosclerosis remains elusive.

Interestingly, the matricellular protein cartilage oligomeric matrix protein (COMP)/thrombospondin-5 inhibits disturbed flow-induced inflammatory EC activation and atherogenesis through inactivating integrin $\alpha 5$, thus preventing $\alpha 5 \beta 1$-fibronectin engagement ( $\mathrm{Lv}$ et al., 2021). COMP deficiency leads to accelerated atherosclerosis, plaque calcification, and post injury restenosis (Wang et al., 2010). The degradation of COMP has been associated with atherosclerosis progression in patients with symptomatic carotid stenosis by measuring circulating levels of COMP and its fragments (Sandstedt et al., 2021). It is worth noting that COMP is mostly secreted by vascular smooth muscle cells in the arterial wall, not by ECs (Fu et al., 2016). 
It is likely that intimal smooth muscle cells are responsible for producing and distributing COMP protein to the basement membrane of endothelium.

\section{DISCUSSION}

Current therapy for atherosclerosis primarily targets hyperlipidemia, thrombosis, and more recently vascular inflammation (Libby and Hansson, 2018). No available treatments directly target the dysfunctional endothelium in atherosclerosis. Patients with the conditions, such as existing atherosclerotic plaque, in-stent restenosis, bypass graft occlusion, transplant vasculopathy, or aortic valve calcification, are prone to further development of atherosclerotic lesion due to endothelial dysfunction caused by disturbed blood flow in the affected vascular segments (Chiu and Chien, 2011). The interaction between ECM and endothelial integrin receptors becomes a favorable therapeutic target for its accessibility and its critical role in atherosclerosis discussed in this review. The caveat of blocking provisional matrix proteins, such as fibronectin, poses a threat of complications because of the diverse and indispensable functions of fibronectin (George et al., 1993; Liu et al., 2010). Alternatively, integrin $\alpha 5$ can be considered as a target. Though $\alpha 5$-null mutation causes embryonic lethality in mice (Yang et al., 1993), $\alpha 5^{+/-}$mice are viable and display significant resistance to disturbed flow-induced EC dysfunction and atherosclerosis (Sun et al., 2016), suggesting a benefit from lowering $\alpha 5$ activities. An integrin $\alpha 5 \beta 1$-blocking peptide (ATN161) successfully abolishes disturbed flow-induced YAP activation and atherogenesis in $A p o e^{-/-}$mice (Li et al., 2019).

\section{REFERENCES}

Al-Yafeai, Z., Yurdagul, A. Jr., Peretik, J. M., Alfaidi, M., Murphy, P. A., and Orr, A. W. (2018). Endothelial FN (Fibronectin) deposition by $\alpha 5 \beta 1$ Integrins drives atherogenic inflammation. Arterioscler. Thromb. Vasc. Biol. 38, 2601-2614. doi: 10.1161/ATVBAHA.118.311705

Bornstein, P., and Sage, E. H. (2002). Matricellular proteins: extracellular modulators of cell function. Curr. Opin. Cell Biol. 14, 608-616. doi: 10.1016/ s0955-0674(02)00361-7

Chatzizisis, Y. S., Jonas, M., Coskun, A. U., Beigel, R., Stone, B. V., Maynard, C., et al. (2008). Prediction of the localization of high-risk coronary atherosclerotic plaques on the basis of low endothelial shear stress: an intravascular ultrasound and histopathology natural history study. Circulation 117, 993-1002. doi: 10.1161/CIRCULATIONAHA.107.695254

Chen, J., Green, J., Yurdagul, A. Jr., Albert, P., Mcinnis, M. C., and Orr, A. W. (2015). alphavbeta3 Integrins mediate flow-induced NF-kappaB activation, proinflammatory gene expression, and early atherogenic inflammation. Am. J. Pathol. 185, 2575-2589. doi: 10.1016/j.ajpath. 2015.05.013

Chen, C. C., and Lau, L. F. (2009). Functions and mechanisms of action of CCN matricellular proteins. Int. J. Biochem. Cell Biol. 41, 771-783. doi: 10.1016/j.biocel.2008.07.025

Chiu, J. J., and Chien, S. (2011). Effects of disturbed flow on vascular endothelium: pathophysiological basis and clinical perspectives. Physiol. Rev. 91, 327-387. doi: 10.1152/physrev.00047.2009

Feaver, R. E., Gelfand, B. D., Wang, C., Schwartz, M. A., and Blackman, B. R. (2010). Atheroprone hemodynamics regulate fibronectin deposition to create positive feedback that sustains endothelial inflammation. Circ. Res. 106, 1703-1711. doi: 10.1161/CIRCRESAHA.109.216283
Additionally, CCN1- $\alpha 6 \beta 1$ engagement is proven a promising therapeutic target for atherosclerosis (Hsu et al., 2019). Because laminin- $\alpha 6 \beta 1$ signaling is important for endothelial homeostasis and function, targeting $\alpha 6 \beta 1$ may be at risk of losing the atheroprotective effect from the native ECM. The antagonistic peptide T1 (derived from an $\alpha 6 \beta 1$-binding sequence of CCN1) preferentially blocks $\alpha 6 \beta 1$ binding with CCN1, without affecting binding with laminin. T1 peptide effectively blocks flowinduced atheroprone activation in ECs and atherogenesis in mice (Hsu et al., 2019). Moreover, a COMP-derived peptidomimetics (CCPep24), designed as an agonist for the specific COMP- $\alpha 5$ interaction, provides atheroprotection against flow-induced EC activation and atherogenesis in mice (Lv et al., 2021). Together, synthetic peptides or peptidomimetics have been successfully used to offer selective inhibition on interactions between specific provisional matrix components and their integrin receptors, and validate the approach targeting the pairing between matrix proteins and integrins for atherosclerosis therapy.

\section{AUTHOR CONTRIBUTIONS}

The author confirms being the sole contributor of this work and has approved it for publication.

\section{FUNDING}

This work was supported by grants MOST 1092320B006044-MY3 from the Ministry of Science and Technology of Taiwan.

Fu, Y., Gao, C., Liang, Y., Wang, M., Huang, Y., Ma, W., et al. (2016). Shift of macrophage phenotype due to cartilage oligomeric matrix protein deficiency drives atherosclerotic calcification. Circ. Res. 119, 261-276. doi: 10.1161/ CIRCRESAHA.115.308021

George, E. L., Georges-Labouesse, E. N., Patel-King, R. S., Rayburn, H., and Hynes, R. O. (1993). Defects in mesoderm, neural tube and vascular development in mouse embryos lacking fibronectin. Development 119, 1079-1091.

Gimbrone, M. A. Jr., and Garcia-Cardena, G. (2016). Endothelial cell dysfunction and the pathobiology of atherosclerosis. Circ. Res. 118, 620-636. doi: 10.1161/ CIRCRESAHA.115.306301

Green, J., Yurdagul, A. Jr., Mcinnis, M. C., Albert, P., and Orr, A. W. (2014). Flow patterns regulate hyperglycemia-induced subendothelial matrix remodeling during early atherogenesis. Atherosclerosis 232, 277-284. doi: 10.1016/j. atherosclerosis.2013.11.052

Hilfiker, A., Hilfiker-Kleiner, D., Fuchs, M., Kaminski, K., Lichtenberg, A., Rothkotter, H. J., et al. (2002). Expression of CYR61, an angiogenic immediate early gene, in arteriosclerosis and its regulation by angiotensin II. Circulation 106, 254-260. doi: 10.1161/01.CIR.0000021426.87274.62

Hsu, P. L., Chen, J. S., Wang, C. Y., Wu, H. L., and Mo, F. E. (2019). Shearinduced CCN1 promotes atheroprone endothelial phenotypes and atherosclerosis. Circulation 139, 2877-2891. doi: 10.1161/CIRCULATIONAHA. 118.033895

Hynes, R. O. (2002). Integrins: bidirectional, allosteric signaling machines. Cell 110, 673-687. doi: 10.1016/S0092-8674(02)00971-6

Kireeva, M. L., Lam, S. C., and Lau, L. F. (1998). Adhesion of human umbilical vein endothelial cells to the immediate-early gene product Cyr61 is mediated through integrin $\alpha \mathrm{v} \beta 3$. J. Biol. Chem. 273, 3090-3096. doi: 10.1074/ jbc.273.5.3090 
Korenaga, R., Ando, J., Kosaki, K., Isshiki, M., Takada, Y., and Kamiya, A. (1997). Negative transcriptional regulation of the VCAM-1 gene by fluid shear stress in murine endothelial cells. Am. J. Phys. 273, C1506-C1515. doi: 10.1152/ajpcell.1997.273.5.c1506

Kundi, R., Hollenbeck, S. T., Yamanouchi, D., Herman, B. C., Edlin, R., Ryer, E. J., et al. (2009). Arterial gene transfer of the TGF-beta signalling protein Smad3 induces adaptive remodelling following angioplasty: a role for CTGF. Cardiovasc. Res. 84, 326-335. doi: $10.1093 / \mathrm{cvr} / \mathrm{cvp} 220$

Lau, L. F. (2016). Cell surface receptors for CCN proteins. J. Cell Commun. Signal. 10, 121-127. doi: 10.1007/s12079-016-0324-Z

Leu, S. J., Liu, Y., Chen, N., Chen, C. C., Lam, S. C., and Lau, L. F. (2003). Identification of a novel integrin alpha 6beta 1 binding site in the angiogenic inducer CCN1 (CYR61). J. Biol. Chem. 278, 33801-33808. doi: 10.1074/jbc. M305862200

Li, B., He, J., Lv, H., Liu, Y., Lv, X., Zhang, C., et al. (2019). c-Abl regulates YAPY357 phosphorylation to activate endothelial atherogenic responses to disturbed flow. J. Clin. Invest. 129, 1167-1179. doi: 10.1172/JCI122440

Libby, P., and Hansson, G. K. (2018). Taming immune and inflammatory responses to treat atherosclerosis. J. Am. Coll. Cardiol. 71, 173-176. doi: 10.1016/j.jacc.2017.10.081

Liu, K., Cheng, L., Flesken-Nikitin, A., Huang, L., Nikitin, A. Y., and Pauli, B. U. (2010). Conditional knockout of fibronectin abrogates mouse mammary gland lobuloalveolar differentiation. Dev. Biol. 346, 11-24. doi: 10.1016/j. ydbio.2010.07.001

Lv, H., Wang, H., Quan, M., Zhang, C., Fu, Y., Zhang, L., et al. (2021). Cartilage oligomeric matrix protein fine-tunes disturbed flow-induced endothelial activation and atherogenesis. Matrix Biol. 95, 32-51. doi: 10.1016/j. matbio.2020.10.003

Orr, A. W., Sanders, J. M., Bevard, M., Coleman, E., Sarembock, I. J., and Schwartz, M. A. (2005). The subendothelial extracellular matrix modulates NF-kappaB activation by flow: a potential role in atherosclerosis. J. Cell Biol. 169, 191-202. doi: 10.1083/jcb.200410073

Sandstedt, J., Vargmar, K., Björkman, K., Ruetschi, U., Bergström, G., Hultén, L. M., et al. (2021). COMP (cartilage oligomeric matrix protein) neoepitope: a novel biomarker to identify symptomatic carotid stenosis. Arterioscler. Thromb. Vasc. Biol. 41, 1218-1228. doi: 10.1161/ATVBAHA.120.314720

Schober, J. M., Chen, N., Grzeszkiewicz, T. M., Jovanovic, I., Emeson, E. E., Ugarova, T. P., et al. (2002). Identification of integrin alpha(M)beta(2) as an adhesion receptor on peripheral blood monocytes for Cyr61 (CCN1) and connective tissue growth factor (CCN2): immediate-early gene products expressed in atherosclerotic lesions. Blood 99, 4457-4465. doi: 10.1182/blood.V99.12.4457

Seano, G., Chiaverina, G., Gagliardi, P. A., Di Blasio, L., Puliafito, A., Bouvard, C., et al. (2014). Endothelial podosome rosettes regulate vascular branching in tumour angiogenesis. Nat. Cell Biol. 16, 931-941. doi: 10.1038/ncb3036

Souilhol, C., Serbanovic-Canic, J., Fragiadaki, M., Chico, T. J., Ridger, V., Roddie, H., et al. (2020). Endothelial responses to shear stress in atherosclerosis: a novel role for developmental genes. Nat. Rev. Cardiol. 17, 52-63. doi: 10.1038/s41569-019-0239-5

Stone, P. H., Saito, S., Takahashi, S., Makita, Y., Nakamura, S., Kawasaki, T., et al. (2012). Prediction of progression of coronary artery disease and clinical outcomes using vascular profiling of endothelial shear stress and arterial plaque characteristics: the PREDICTION study. Circulation 126, 172-181. doi: 10.1161/CIRCULATIONAHA.112.096438
Stupack, D. G., and Cheresh, D. A. (2002). ECM remodeling regulates angiogenesis: endothelial integrins look for new ligands. Sci. STKE 2002:E7. doi: 10.1126/ stke.2002.119.pe7

Sun, X., Fu, Y., Gu, M., Zhang, L., Li, D., Li, H., et al. (2016). Activation of integrin $\alpha 5$ mediated by flow requires its translocation to membrane lipid rafts in vascular endothelial cells. Proc. Natl. Acad. Sci. U. S. A. 113, 769-774. doi: $10.1073 /$ pnas. 1524523113

Wang, L., Luo, J. Y., Li, B., Tian, X. Y., Chen, L. J., Huang, Y., et al. (2016b). Integrin-YAP/TAZ-JNK cascade mediates atheroprotective effect of unidirectional shear flow. Nature 540, 579-582. doi: 10.1038/nature20602

Wang, K. C., Yeh, Y. T., Nguyen, P., Limqueco, E., Lopez, J., Thorossian, S., et al. (2016a). Flow-dependent YAP/TAZ activities regulate endothelial phenotypes and atherosclerosis. Proc. Natl. Acad. Sci. U. S. A. 113, 11525-11530. doi: $10.1073 /$ pnas. 1613121113

Wang, L., Zheng, J., Du, Y., Huang, Y., Li, J., Liu, B., et al. (2010). Cartilage oligomeric matrix protein maintains the contractile phenotype of vascular smooth muscle cells by interacting with alpha(7)beta(1) integrin. Circ. Res. 106, 514-525. doi: 10.1161/CIRCRESAHA.109.202762

Yang, J. T., Rayburn, H., and Hynes, R. O. (1993). Embryonic mesodermal defects in alpha 5 integrin-deficient mice. Development 119, 1093-1105.

Yu, F. X., Zhao, B., and Guan, K. L. (2015). Hippo pathway in organ size control, tissue homeostasis, and cancer. Cell 163, 811-828. doi: 10.1016/j. cell.2015.10.044

Yun, S., Hu, R., Schwaemmle, M. E., Scherer, A. N., Zhuang, Z., Koleske, A. J., et al. (2019). Integrin $\alpha 5 \beta 1$ regulates PP2A complex assembly through PDE4D in atherosclerosis. J. Clin. Invest. 129, 4863-4874. doi: 10.1172/JCI127692

Yurchenco, P. D., and O'Rear, J. J. (1994). Basal lamina assembly. Curr. Opin. Cell Biol. 6, 674-681. doi: 10.1016/0955-0674(94)90093-0

Yurdagul, A. Jr., Finney, A. C., Woolard, M. D., and Orr, A. W. (2016). The arterial microenvironment: the where and why of atherosclerosis. Biochem. J. 473, 1281-1295. doi: 10.1042/BJ20150844

Yurdagul, A. Jr., Green, J., Albert, P., Mcinnis, M. C., Mazar, A. P., and Orr, A. W. (2014). alpha5betal integrin signaling mediates oxidized low-density lipoprotein-induced inflammation and early atherosclerosis. Arterioscler. Thromb. Vasc. Biol. 34, 1362-1373. doi: 10.1161/ATVBAHA.114.303863

Zhang, C., Zhou, T., Chen, Z., Yan, M., Li, B., Lv, H., et al. (2020). Coupling of integrin $\alpha 5$ to annexin A2 by flow drives endothelial activation. Circ. Res. 127, 1074-1090. doi: 10.1161/CIRCRESAHA.120.316857

Zhu, J. J., Jiang, Z. T., Liu, C., Xi, Y. F., Wang, J., Yang, F. F., et al. (2020). VAMP3 and SNAP23 as potential targets for preventing the disturbed flowaccelerated thrombus formation. Front. Cell Dev. Biol. 8:576826. doi: 10.3389/ fcell.2020.576826

Conflict of Interest: The author declares that the research was conducted in the absence of any commercial or financial relationships that could be construed as a potential conflict of interest.

Copyright (C) 2021 Mo. This is an open-access article distributed under the terms of the Creative Commons Attribution License (CC BY). The use, distribution or reproduction in other forums is permitted, provided the original author(s) and the copyright owner(s) are credited and that the original publication in this journal is cited, in accordance with accepted academic practice. No use, distribution or reproduction is permitted which does not comply with these terms. 